\title{
AVERAGE CO-ORDINATE ENTROPY
}

\author{
GENEVIEVE MORTISS
}

(Received 15 December 2000; revised 28 August 2001)

Communicated by A. H. Dooley

\begin{abstract}
A notion of entropy for the non-singular action of finite co-ordinate changes on $\left(X=\prod_{i=1}^{\infty} \mathbb{Z}_{2}, \mu\right)$ is introduced. This quantity - average co-ordinate or AC entropy-is calculated for product measures and $G$-measures. It is shown that the type III classes can be subdivided using AC entropy. An equivalence relation is established for which AC entropy is an invariant.
\end{abstract}

2000 Mathematics subject classification: primary 28D20, $28 \mathrm{~A} 35$.

\section{Introduction}

Let $T$ be a measure preserving transformation on the space $(X, \mathscr{B}, \mu)$ and $\alpha$ be a finite, measurable partition of $X$. Then the entropy of $T$ with respect to $\alpha$ is given by the formula

$$
h(T, \alpha)=\lim _{n \rightarrow \infty} \frac{1}{n} H\left(\bigvee_{i=0}^{\infty} T^{-i} \alpha\right),
$$

where $H(\beta)=-\sum_{i=1}^{n} \mu\left(B_{i}\right) \log \mu\left(B_{i}\right)$ for $\beta=\left\{B_{i}\right\}_{i=1}^{n}$ a finite measurable partition of $X$. The entropy of $T$ is defined as $h(T)=\sup _{\alpha} h(T, \alpha)$.

Now the above limit must always exist for $T$ measure preserving, and further the entropy of a transformation is invariant for metric isomorphism. Both these results rely upon the fact that $H\left(\phi^{-1} \alpha\right)=H(\alpha)$ for any measure preserving automorphism $\phi$. Obviously this is not necessarily true for a non-singular $\phi$.

Attempts have been made to define a non-singular version of entropy. For example in [12], an entropy is defined using the Krengel entropy of skew products. However the resulting quantity is somewhat limited as it only takes the values zero and infinity.

(C) 2002 Australian Mathematical Society $1446-7887 / 2000 \$ A 2.00+0.00$ 
In this paper we formulate a new notion of entropy for a class of non-singular dynamical systems which have been the subject of much recent interest-systems of finite co-ordinate changes. This new quantity, which we will call average co-ordinate or AC entropy may be regarded as a measure of the average uncertainty or randomness in the initial co-ordinates of a point $x \in X=\prod_{i=1}^{\infty} \mathbb{Z}_{2}$ as it is acted upon by the group

$$
\Gamma=\left\{\gamma \in X: \exists N \in \mathbb{N} \text { such that } \gamma_{n}=0 \text { for } n>N\right\} .
$$

Our original motivation for examining such systems arose from their relative simplicity. Having the same group acting on two spaces $(X, \mathscr{B}, \mu)$ and $(X, \mathscr{B}, \nu)$ means that we need only attend to the differences in their measures.

We begin with some notation before we give our definition of AC entropy. When we write $(X, \Gamma, \mu)$ we are denoting the system of finite co-ordinate changes that is the action of $\Gamma$ on $(X, \mathscr{B}, \mu)$. Here $\mu$ is a probability measure on $X=\prod_{i=1}^{\infty} \mathbb{Z}_{2}$ which is quasi-invariant and ergodic for the action of $\Gamma$. For an element $\gamma \in \Gamma$, $\gamma x \in X$ is defined by $(\gamma x)_{n}=\gamma_{n}+x_{n}(\bmod 2)$. Let $\Gamma_{n}$ denote the subgroup $\left\{\gamma \in \Gamma: \gamma_{k}=0\right.$ for all $\left.k>n\right\}$ and $X_{n}$ the set $\left\{x \in X: x_{1}=x_{2}=\cdots=x_{n}=0\right\}$.

Note that while we have chosen to work with $X=\prod_{i=1}^{\infty} \mathbb{Z}_{2}$, we could easily extend our work to cover $X=\prod_{i=1}^{\infty} \mathbb{Z}_{p}$ for any $p \in \mathbb{N}$.

The real differences between two systems of finite co-ordinate changes lie in their measures. Therefore we denote our new entropy as $h_{\mathrm{AC}}(\mu)$ where $\mu$ is the measure on the system under consideration.

For all partitions $\zeta$ and $\eta$ if $\zeta \leq \eta$ then $H(\zeta) \leq H(\eta)$. If our new entropy is to be similar in some respect to the standard form then we should be looking for some kind of supremum over partitions. For this reason we define AC entropy using $\beta_{n}=\left\{\gamma X^{n}\right\}_{\gamma \in \Gamma_{n}}$ the finest cylinder set partition for fixed $n \in \mathbb{N}$.

DEFINITION 1.1. For a system of finite co-ordinate changes $(X, \Gamma, \mu)$ we define the AC entropy by

$$
h_{\mathrm{AC}}(\mu)=\lim _{n \rightarrow \infty} \frac{1}{n} H\left(\beta_{n}\right)
$$

where this exists. If this limit does not exist we say that the $\mathrm{AC}$ entropy of $\mu$ is undefined.

Here the prefix AC stands for 'average co-ordinate', and will be used to distinguish our new quantity from the standard metric entropy.

Note that our definition maintains the same basic entropy ratio as we find in the metric entropy of a transformation acting on $\alpha$ a two set partition of $X$. For fixed $n$, the maximum number of non-trivial elements in $\bigvee_{i=0}^{n-1} T^{-i}(\alpha)$ is $2^{n}$, the same as the number of elements in $\beta_{n}$. 
We provide formulae for the AC entropy for two important classes of measuresnamely Markov measures (a subclass of Brown and Dooley's $G$-measures introduced in [1]) and product measures. In the latter case AC entropy reduces to an extremely simple expression. It is for this reason that much of our focus will be on product measures, although in the longer term we hope to extend all our results to $G$-measures. We also give results relating to $\mathrm{AC}$ entropy and measure equivalence. It follows from Kakutani's theorem that two equivalent product measures have the same AC entropy. While we cannot make the same statement for Markov measures generally, we are able to produce such a result for a special type of these measures which we have called Quas-Markov.

In Section 4 we show that AC entropy can be used to subdivide the type III classes. The AC entropy of a system, if it exists, lies in the interval $[0,1]$. For each $\lambda \in[0,1]$ there exist type III $_{\lambda}$ systems of all possible AC entropies.

Ultimately our aim in this work would be to prove a result analogous to Ornstein's isomorphism theorem using AC entropy as an invariant. To this end in Section 5 we define an equivalence relation which we shall call Initial Co-ordinate or IC equivalence. Roughly speaking, two systems $(X, \Gamma, \mu)$ and $(X, \Gamma, \nu)$ are IC equivalent if there exists a suitable map between them which preserves equality of initial co-ordinates between two points. We give some examples of IC equivalences and show that two IC equivalent systems have the same AC entropy. Further work may allow us to define a class of systems within which AC entropy would be a complete invariant for IC equivalence, as entropy is a complete invariant for isomorphism between finitely determined systems.

Recently it has been discovered that the AC entropy of a system of finite coordinate changes $(X, \Gamma, \mu)$ is closely related to another invariant known as the critical dimension associated with the odometer action on $(X, \mu)$. The critical dimension ([10]) quantifies the asymptotic growth rate of the Radon derivative sums $\sum_{i=0}^{n-1} d T^{-i} \mu(x) / d \mu$ and is an invariant for isomorphism of non-singular ergodic transformations. The relationship between the AC entropy of a system $(X, \Gamma, \mu)$ for $\mu$ a product measure and the critical dimension of the associated odometer can be appreciated by considering a Shannon-McMillan-Breiman type theorem in Section 2. By this theorem, the results of Section 4 have immediate implications for the study of non-singular isomorphism classes.

\section{Product measures}

Let $\mu=\bigotimes_{i=1}^{\infty} \mu_{i}$ be a product measure on $X$ with

$$
\mu_{i}(1)=\left(1+a_{i}\right) / 2, \quad \mu_{i}(0)=\left(1-a_{i}\right) / 2
$$

where $a_{i} \in(-1,1)$. Then by an easy calculation we have: 
PROPOSITION 2.1. For a product measure as described

$$
h_{\mathrm{AC}}(\mu)=\lim _{n \rightarrow \infty} \frac{-1}{n} \sum_{i=1}^{n} \frac{1+a_{i}}{2} \log \frac{1+a_{i}}{2}+\frac{1-a_{i}}{2} \log \frac{1-a_{i}}{2} .
$$

NOTE. Unless otherwise stated, all logs are taken to base 2.

This formula will feature in much of the remainder of this paper.

If $\mu=\bigotimes_{i=1}^{\infty} \mu_{i}, \nu=\bigotimes_{i=1}^{\infty} \nu_{i}$ are two infinite product measures on $X=\prod_{i=1}^{\infty} \mathbb{Z}_{2}$, then by Kakutani's Theorem we can state the following fact:

PROPOSTTION 2.2. If $\mu$ and $\nu$ are equivalent measures on $X$ then $h_{\mathrm{AC}}(\mu)=h_{\mathrm{AC}}(\nu)$.

But of course it is not hard to see that there exist non-equivalent measures with the same AC entropy.

EXAMPLE. Let $\mu_{i}(0)=a, \mu_{i}=(1)=1-a$ for $i$ odd while $\mu_{i}(0)=b, \mu_{i}(1)=$ $1-b$ for $i$ even where $a \neq b$. Then take $v_{i}(0)=b, v_{i}(1)=1-b$ for $i$ odd and $v_{i}(0)=a, v_{i}(1)=1-a$ for $i$ even.

The above example leads us to consider invariance of AC entropy in permuted measures. That is, if $\nu_{i}=\mu_{\pi(i)}$ for what sort of permutations $\pi$ do we have $h_{\mathrm{AC}}(v)=$ $h_{\mathrm{AC}}(\mu)$ ? We can at least partly answer this question.

PROPOSITION 2.3. Suppose $h_{\mathrm{AC}}(\mu)$ exists and $\pi$, a permutation of $\mathbb{N}$ satisfies

$$
\lim _{n \rightarrow \infty} \frac{\#|\pi:(1, n) \Delta(1, n)|}{n}=0 .
$$

(Here $\pi:(1, n)$ is the range of $\pi$ over $(1, n)$.) Then if $\nu_{i}=\mu_{\pi(i)}$ then $h_{\mathrm{AC}}(v)$ exists and equals $h_{\mathrm{AC}}(\mu)$.

Note that the set of permutations $\pi$ satisfying (1) forms a subgroup under composition.

We include the following example to illustrate how the previous result may fail without the limit condition on $\pi$.

EXAMPLE. Define $\mu=\bigotimes_{i=1}^{\infty} \mu_{i}$ on $X$ such that

$$
H\left(\mu_{i}\right)= \begin{cases}1, & \text { for } i \text { odd } \\ 1 / 2, & \text { for } i \text { even }\end{cases}
$$

So $h_{\mathrm{AC}}(\mu)=1 / 2+1 / 2 \cdot 1 / 2=3 / 4$. Now let $\nu=\bigotimes_{i=1}^{\infty} \nu_{i}=\bigotimes_{i=1}^{\infty} \mu_{\pi(i)}$ where $\pi$ is defined as follows: 
Divide $\mathbb{N}$ into blocks $\{1\},\{2,3\},\{4,5,6,7\}, \ldots,\left\{2^{n}, \ldots, 2^{n+1}-1\right\}, \ldots$ Let $\pi$ be a permutation that sends all the even integers in $\left\{2^{n}, \ldots, 2^{n+1}-1\right\}$ to the first half of that block and all the odd integers to the second half. For example, such a $\pi$ acting on the block $\{4,5,6,7\}$ could give us $\{4,6,5,7\}$.

Now at the end of each block, that is, at $i=2^{n}-1$

$$
\frac{\#\left|\left(1,2^{n}-1\right) \Delta \pi:\left(1,2^{n}-1\right)\right|}{2^{n}-1}=0
$$

and

$$
\lim _{n \rightarrow \infty} \frac{1}{2^{n}-1} \sum_{i=1}^{2^{n}-1} H\left(\mu_{i}\right)=\frac{3}{4}=\lim _{n \rightarrow \infty} \frac{1}{2^{n}-1} \sum_{i=1}^{2^{n}-1} H\left(v_{i}\right) .
$$

But in the middle of each block, that is, at $i=2^{n}+2^{n-1}-1$,

$$
\frac{\#\left|\left(1,2^{n}+2^{n-1}-1\right) \Delta \pi:\left(1,2^{n}+2^{n-1}-1\right)\right|}{2^{n}+2^{n-1}-1} \geq \frac{2^{n-2}}{2^{n}+2^{n-1}-1} \rightarrow \frac{1}{6} \quad \text { as } n \rightarrow \infty
$$

and

$$
\frac{1}{2^{n}+2^{n-1}-1} \sum_{i=1}^{2^{n}+2^{n-1}-1} H\left(v_{i}\right)=\frac{1}{2^{n}+2^{n-1}-1}\left(\frac{2^{n}}{2} \frac{1}{2}+\frac{2^{n}}{2}+\frac{2^{n-1}-1}{2}\right) \rightarrow \frac{2}{3}\left(\neq \frac{3}{4}\right)
$$

as $n \rightarrow \infty$. Thus the AC entropy of $v$ is not defined. So even the existence of the AC entropy limit is not preserved under $\pi$.

To conclude this section we present the following Shannon-McMillan-Breiman type result:

THEOREM 2.4. Let $X$ be equipped with a product measure $\mu=\bigotimes_{i=1}^{\infty} \mu_{i}$. Then

$$
\lim _{n \rightarrow \infty} \frac{\sum_{i=1}^{n}-\log \left(\mu_{i}\left(x_{i}\right)\right)+\mu_{i}(0) \log \left(\mu_{i}(0)\right)+\mu_{i}(1) \log \left(\mu_{i}(1)\right)}{n}=0
$$

for $\mu$-almost all $x \in X$.

The proof Theorem 2.4 is easily derived from the following result from [4].

THEOREM 2.5. Let $y_{1}, y_{2}, \ldots$ be mutually independent random variables with $E\left(y_{i}\right)=0$ and $E\left(y_{i}^{2}\right)=\sigma_{i}^{2}<\infty$. Then if $\sum_{1}^{\infty}\left(\sigma_{i}^{2} / i^{2}\right)<\infty$, we have

$$
\lim _{n \rightarrow \infty} \frac{y_{1}+\cdots+y_{n}}{n}=0
$$

for $\mu$-almost all $x \in X$. 
Note that it has been shown ([10]) that the critical dimension of a odometer is equal to $\lim \inf _{n \rightarrow \infty}(1 / n) \sum_{i=1}^{n}-\log \left(\mu_{i}\left(x_{i}\right)\right)$ for $\mu$-almost all $x \in X$ for a wide class of product measures. Further, it can be established that for any product measures if the AC entropy limit exists then it is equal to the critical dimension. In light of this it may seem tempting to have begun our study of AC entropy by defining it as $\liminf _{n \rightarrow \infty}(1 / n) H\left(\beta_{n}\right)$ expecting that for a general measure on $X$ the critical dimension of the associated odometer action would be the same. However, as will be shown in the following section, a question remains as to whether two equivalent Markov odometers (which must have the same critical dimension) can have different AC entropies. Hence it would be premature at this stage to try and identify the two invariants, although it is fairly clear that some close relationship does exist in the general case.

\section{3. $G$-measures}

Brown and Dooley's $G$-measures [1] are a generalized form of Keane's $g$-measures [6]. Calculating the AC entropy of a general $G$-measure (Proposition 3.4) does not really tell us anything concrete and for this reason we narrow our focus to Markov measures. Within a restricted class of these measures, which we shall refer to as the Quas-Markov class, we are able to show that provided two measures satisfy the hypotheses of the Kakutani's Theorem analogue for $G$-measures then AC entropy is an invariant for measure equivalence. However, as we explain later in this section, the same relationship may not hold for general Markov measures.

DEFINITION 3.1. Let $G=\left(G_{n}\right)_{n=1}^{\infty}$ be a family of non-negative Borel functions on $X=\prod_{i=1}^{\infty} \mathbb{Z}_{2}$ satisfying

(i) normalization, that is, $\left(1 /\left|\Gamma_{n}\right|\right) \sum_{\gamma \in \Gamma_{n}} G_{n}(\gamma x)=1$ for all $x \in X$; and

(ii) compatibility, that is, $G_{n}(\gamma x) G_{m}(x)=G_{m}(\gamma x) G_{n}(x)$, where $n>m, \gamma \in \Gamma_{m}$ and $x \in X$.

Then from this family of functions we construct the family $\left(g_{n}(x)\right)_{n=1}^{\infty}$, where

$$
g_{n}(x)= \begin{cases}G_{n}(x) / G_{n-1}(x), & G_{n-1}(x) \neq 0 \\ 0, & G_{n-1}(x)=0\end{cases}
$$

which satisfy the following

(i) $g_{n}(x)$ depends only on the co-ordinates $\left(x_{n}, x_{n+1}, \ldots\right)$; and

(ii) for each $n \in \mathbb{N},\left(g_{n}\left(0, x_{n+1}, x_{n+2}, \ldots\right)+g_{n}\left(1, x_{n+1}, x_{n+2}, \ldots\right)\right) / 2=1$ for all $x \in X$. 
Now we say $\mu$ is a $G$-measure if $d \mu(x) / d \mu^{(n)}=\prod_{i=1}^{n} g_{i}(x)=G_{n}(x)$ where $\mu^{(n)}=$ $\left(1 /\left|\Gamma_{n}\right|\right) \sum_{\gamma \in \Gamma_{n}} \mu \circ \gamma$.

Our work in this section relies upon the following two results. The first is necessary for our AC entropy calculations (Proposition 3.4 and Proposition 3.5). The second is a $G$-measure version of Kakutani's Theorem which we require for the proof of Lemma 3.7.

LEMMA 3.2 ([1]). Let $\mu$ be a quasi-invariant $G$-measure. Then for each $k \in \mathbb{N}$, $g_{k}(x)>0$ for $\mu$ a.a. $x \in X$, and for $\gamma \in \Gamma$,

$$
\frac{d \mu \circ \gamma}{d \mu}(x)=\prod_{k=1}^{\infty} \frac{g_{k}(\gamma x)}{g_{k}(x)} .
$$

LEMMA 3.3 ([2]). Suppose $\mu$ is a unique G-measure and $\nu$ is a unique $F$-measure and that if $g_{i}(x)=0$, then $f_{i}(x)=0$. Then the following conditions are equivalent:

(i) $\mu \sim \nu$;

(ii) the series $\sum_{n=1}^{\infty} \sum_{\gamma \in \mathbb{Z}_{2}}\left(\sqrt{f_{n}(\gamma x)}-\sqrt{g_{n}(\gamma x)}\right)^{2}$ converges for $v$ almost all $x$;

(iii) the above series converges on a set of positive v-measure.

PROPOSITION 3.4. For a quasi-invariant G-measure

$$
\begin{aligned}
H\left(\beta_{n}\right) & =-\sum_{\gamma \in \Gamma_{n}} \mu\left(\gamma X^{n}\right) \log \mu\left(\gamma X^{n}\right) \\
& =-\sum_{\gamma \in \Gamma_{n}} \int_{X^{n}} \frac{d \mu \circ \gamma}{d \mu}(x) d \mu(x) \log \left(\int_{X^{n}} \frac{d \mu \circ \gamma}{d \mu}(x) d \mu(x)\right) \\
& =-\sum_{\gamma \in \Gamma_{n}} \int_{X^{n}} \prod_{k=1}^{n} \frac{g_{k}(\gamma x)}{g_{k}(x)} d \mu(x) \log \left(\int_{X^{n}} \prod_{k=1}^{n} \frac{g_{k}(\gamma x)}{g_{k}(x)} d \mu(x)\right) \\
& =-\sum_{\gamma \in \Gamma_{n}} \int_{X^{n}} \prod_{k=1}^{n} g_{k}(\gamma x) d \mu^{(n)}(x) \log \left(\int_{X^{n}} \prod_{k=1}^{n} g_{k}(\gamma x) d \mu^{(n)}(x)\right) .
\end{aligned}
$$

Unfortunately this is about as far as we can go without making assumptions about the functions $g_{k}(x)$. For this reason we shall restrict ourselves to considering Markov measures, that is, those $G$-measures for which $g_{n}(x)=g_{n}\left(x_{n}, x_{n+1}\right)$. So our normalization condition reduces to $\left(g_{n}\left(0, x_{n+1}\right)+g_{n}\left(1, x_{n+1}\right)\right) / 2=1$. Now

$$
H\left(\beta_{n}\right)=-\sum_{\gamma \in \Gamma_{n}} \mu\left(\gamma X^{n}\right) \log \mu\left(\gamma X^{n}\right),
$$


so first we will need a formula for $\mu\left(\gamma X^{n}\right)$, where $\gamma \in \Gamma_{n}$ and $X^{n}=\left\{x: x_{1}=x_{2}=\right.$ $\cdots=x_{n}=0$ ).

Now if $\gamma \in \Gamma_{n-1}$, we have

$$
\begin{aligned}
\mu\left(\gamma X^{n}\right) & =\int_{X^{n}} \prod_{k=1}^{n-2} \frac{g_{k}\left(\gamma_{k}, \gamma_{k+1}\right)}{g_{k}(0,0)} \frac{g_{n-1}\left(\gamma_{n-1}, 0\right)}{g_{n-1}(0,0)} d \mu \\
& =\prod_{k=1}^{n-2} \frac{g_{k}\left(\gamma_{k}, \gamma_{k+1}\right)}{g_{k}(0,0)} \frac{g_{n-1}\left(\gamma_{n-1}, 0\right)}{g_{n-1}(0,0)} \mu\left(X^{n}\right) .
\end{aligned}
$$

If $\gamma \in \Gamma_{n} \backslash \Gamma_{n-1}$,

$$
\begin{aligned}
\mu\left(\gamma X^{n}\right) & =\int_{X^{n}} \prod_{k=1}^{n-2} \frac{g_{k}\left(\gamma_{k}, \gamma_{k+1}\right)}{g_{k}(0,0)} \frac{g_{n-1}\left(\gamma_{n-1}, 1\right)}{g_{n-1}(0,0)} \frac{g_{n}\left(1, x_{n+1}\right)}{g_{n}\left(0, x_{n+1}\right)} d \mu \\
& =\prod_{k=1}^{n-1} \frac{g_{k}\left(\gamma_{k}, \gamma_{k+1}\right)}{g_{k}(0,0)} \int_{X^{n}} \frac{g_{n}\left(1, x_{n+1}\right)}{g_{n}\left(0, x_{n+1}\right)} d \mu \\
& =\prod_{k=1}^{n-1} \frac{g_{k}\left(\gamma_{k}, \gamma_{k+1}\right)}{g_{k}(0,0)}\left[\frac{g_{n}(1,0)}{g_{n}(0,0)} \mu\left(X^{n+1}\right)+\frac{g_{n}(1,1)}{g_{n}(0,1)}\left(\mu\left(X^{n}\right)-\mu\left(X^{n+1}\right)\right)\right] .
\end{aligned}
$$

Of course these expressions for $\mu\left(\gamma X^{n}\right)$ depend on the values $\mu\left(X^{n}\right)$ and so by using the recursive relationship

$$
\mu\left(X^{n+1}\right)=\left(\frac{\prod_{k=1}^{n-1} g_{k}(0,0)}{2^{n-1}}-\frac{2}{g_{n}(0,1)} \mu\left(X^{n}\right)\right) /\left(\frac{g_{n}(1,0)}{g_{n}(0,0)}-\frac{g_{n}(1,1)}{g_{n}(0,1)}\right)
$$

and some further straightforward calculations we obtain the following:

PROPOSITION 3.5. For a Markov measure $\mu$,

$$
\begin{aligned}
h_{\mathrm{AC}}(\mu)= & \lim _{n \rightarrow \infty} \frac{1}{n}\left[\frac{2^{n-1}}{\prod_{k=1}^{n-1} g_{k}(0,0)}\left(h\left(\mu\left(X^{n}\right)\right)+h\left(\frac{\prod_{k=1}^{n-1} g_{k}(0,0)}{2^{n-1}}-\mu\left(X^{n}\right)\right)\right)\right. \\
& +\mu\left(X^{n}\right) \sum_{\gamma \in \Gamma_{n-1}} h\left(\prod_{k=1}^{n-1} \frac{g_{k}\left(\gamma_{k}, \gamma_{k+1}\right)}{g_{k}(0,0)}\right) \\
& \left.+\left(\frac{\prod_{k=1}^{n-1} g_{k}(0,0)}{2^{n-1}}-\mu\left(X^{n}\right)\right) \sum_{\gamma \in \Gamma_{n} \backslash \Gamma_{n-1}} h\left(\prod_{k=1}^{n-1} \frac{g_{k}\left(\gamma_{k}, \gamma_{k+1}\right)}{g_{k}(0,0)}\right)\right]
\end{aligned}
$$

if this exists, where $h(x)=-x \log x$ and

$$
\mu\left(X^{n}\right)=A_{n-1}+\sum_{k=1}^{n-2}\left(\prod_{i=1}^{n-k} B_{n-i}\right) A_{n-k-1}+\mu\left(X^{1}\right) \prod_{i=1}^{n-1} B_{n-i},
$$


for

$$
A_{n-1}=\frac{\prod_{k=1}^{n-2} g_{k}(0,0)}{2^{n-2}\left(\frac{g_{n-1}(1,0)}{g_{n-1}(0,0)}-\frac{g_{n-1}(1,1)}{g_{n-1}(0,1)}\right)}, \quad B_{n-1}=\frac{-2}{g_{n-1}(0,1)\left(\frac{g_{n-1}(1,0)}{g_{n-1}(0,0)}-\frac{g_{n-1}(1,1)}{g_{n-1}(0,1)}\right)} .
$$

Obviously our formula for AC entropy on even the simplest $G$-measure is extremely cumbersome. However for a certain class of Markov measures discussed in [5] we can obtain a more usable expression.

DEFINITION 3.6. We call $\mu$ on $X=\prod_{i=0}^{\infty} \mathbb{Z}_{2}$ a Quas-Markov measure if there exist $K_{0}, K_{1}=1-K_{0}$ and $P_{i, j}^{(n)}, i, j \in\{0,1\}$, for $n \geq 1$ such that

$$
\mu\left(\left[x_{1}, x_{2}, \ldots, x_{n}\right]\right)=K_{x_{1}} P_{x_{1}, x_{2}}^{(1)} P_{x_{2}, x_{3}}^{(2)} \cdots P_{x_{n-1}, x_{n}}^{n-1}
$$

for $P_{1,1}^{(n)}=P_{0,0}^{(n)}=1-P_{0,1}^{(n)}=1-P_{1,0}^{(n)}$.

The symmetric nature of these measures means that they are product-like in some respects. In [5] Quas-Markov measures were shown to be orbit equivalent to product measures, as their associated flows have the AT property.

LEMMA 3.7. If $\mu$ and $v$ are Quas-Markov measures satisfying the hypotheses of Lemma 3.3 and $\mu \sim \nu$ then if $h_{\mathrm{AC}}(\mu)$ exists then $h_{\mathrm{AC}}(\nu)$ exists and equals $h_{\mathrm{AC}}(\mu)$.

PROOF. Consider the function $\phi:(X, \eta) \rightarrow(X, \mu)$ where $\phi(x)=y$ for $y_{n}=$ $\sum_{i=0}^{n} x_{i}(\bmod 2)$ and $\eta$ is the product measure $\eta=\bigotimes_{i=0}^{\infty} \eta_{i}$ where $\eta_{0}(0)=K_{0}$ and $\eta_{i}(0)=P_{0,0}^{(i)}$ and $\eta_{i}(1)=P_{0,1}^{(i)}$.

As $\phi$ is measure preserving we know that $h_{\mathrm{AC}}(\mu)=h_{\mathrm{AC}}(\eta)$. Now as

and

$$
\frac{K_{1}}{K_{0}} \frac{P_{1,0}^{(n-1)}}{P_{0,0}^{(n-1)}}=\frac{g_{1}(1,1)}{g_{1}(0,0)} \frac{g_{2}(1,1)}{g_{2}(0,0)} \cdots \frac{g_{n-1}(1,0)}{g_{n-1}(0,0)}
$$

$$
\frac{K_{1}}{K_{0}} \frac{P_{1,1}^{(n-1)}}{P_{0,1}^{(n-1)}}=\frac{g_{1}(1,1)}{g_{1}(0,0)} \frac{g_{2}(1,1)}{g_{2}(0,0)} \cdots \frac{g_{n-1}(1,1)}{g_{n-1}(0,1)}
$$

we obtain

$$
\frac{P_{1,1}^{(n-1)}}{P_{0,1}^{(n-1)}} \frac{P_{0,0}^{(n-1)}}{P_{1,0}^{(n-1)}}=\frac{g_{n-1}(1,1)}{g_{n-1}(0,1)} \frac{g_{n-1}(0,0)}{g_{n-1}(1,0)}
$$

As $P_{1,0}^{(n-1)}=1-P_{1,1}^{(n-1)}$ we have

$$
P_{0,1}^{(n-1)}=1 /\left(1+\sqrt{\frac{g_{n-1}(1,1)}{g_{n-1}(0,1)} \frac{g_{n-1}(0,0)}{g_{n-1}(1,0)}}\right) .
$$

So by Lemma 3.3 we have our result. 
The AC entropy of a Quas-Markov measure is easily calculated. For a general Markov measure of the form $\mu\left(\left[x_{1}, x_{2}, \ldots, x_{n}\right]\right)=K_{x_{1}} P_{x_{1}, x_{2}}^{(1)} P_{x_{2}, x_{3}}^{(2)} \cdots P_{x_{n-1}, x_{n}}^{n-1}$ we have the recursive relationship

$$
\begin{aligned}
H\left(\beta_{n}\right)= & H\left(\beta_{n-1}\right)+\mu\left(X_{n-1}^{0}\right)\left(h\left(P_{0,0}^{(n-1)}\right)+h\left(P_{0,1}^{(n-1)}\right)\right) \\
& +\mu\left(X_{n-1}^{1}\right)\left(h\left(P_{1,0}^{(n-1)}\right)+h\left(P_{1,1}^{(n-1)}\right)\right),
\end{aligned}
$$

where $\mu\left(X_{n-1}^{0}\right)$ is the measure of the set of $x \in X$ with $x_{n-1}=0$. This formula will allow us to explain why it may be possible for two equivalent Markov measures to have different AC entropies.

Now by results of Lodkin ([8]) and LePage and Mandrekar ([7]), two Markov measures which are ergodic for $\Gamma$ on $X=\prod_{i=0}^{\infty} \mathbb{Z}_{2}$ and have weights $P_{i, j}^{(n)}, Q_{i, j}^{(n)}$ bounded away from zero are equivalent if and only if

$$
\sum_{i=1}^{\infty}\left(\sqrt{P_{i, j}^{(n)}}-\sqrt{Q_{i, j}^{(n)}}\right)^{2}<\infty
$$

for all $i, j=0,1$. By this condition $\lim _{n \rightarrow \infty} h\left(P_{i, j}^{(n)}\right)=\lim _{n \rightarrow \infty} h\left(Q_{i, j}^{(n)}\right)$. However, it is not immediately clear to us that this condition is sufficient to guarantee equality of AC entropy. In addition, questions remain as to the validity of the Markov measure equivalence criteria if the weights are not bounded away from zero, or if AC entropy is defined on a more general space $X=\prod_{i=1}^{\infty} \mathbb{Z}_{l(i)}$. Hence we leave examination of the detail of this point to a future paper.

\section{AC entropy within orbit equivalence classes}

In the study of ergodic dynamical systems most attention has been given to those systems which are measure preserving. For this reason we believe that in developing a new invariant we should hope to provide more information about the type III systems rather than the already well-studied measure preserving systems and their close relatives in the $\mathrm{II}_{1}$ class.

AC entropy is not an invariant of orbit equivalence as shown by the results below. However it does allow us to subdivide the type III orbit equivalence classes, and so it may hold potential in the investigation of the $\mathrm{III}_{0}$ classes.

In this section we are dealing exclusively with product measures on $X=\prod_{i=1}^{\infty} \mathbb{Z}_{2}$. We summarize our results as follows:

- All $\mathrm{II}_{1}$ systems have $h_{\mathrm{AC}}(\mu)=1$.

- For every $c \in[0,1]$ there is a $\operatorname{III}_{\lambda}(0 \leq \lambda \leq 1)$ system with $h_{\mathrm{AC}}(\mu)=c$.

One of our major tools in this section will be the following result. 
THEOREM 4.1 (Moore's Criteria [9]). Let $\mu$ be a product measure on $X$. Then

(1) $\mu$ is type I if and only if $\sum_{i}\left(1-a_{i}\right)<\infty$;

(2) $\mu$ is type $\mathrm{II}_{1}$ if and only if $\sum_{i} a_{i}^{2}<\infty$;

(3) $\mu$ is type III if and only if $\sum_{i}\left(1-a_{i}\right)\left(\min \left(2 a_{i} /\left(1-a_{i}\right), 1\right)\right)^{2}=\infty$;

(4) if $\mu$ satisfies none of the above conditions then $\mu$ is of type $\mathrm{II}_{\infty}$.

From part (2) it immediately follows that

PROPOSITION 4.2. All $\mathrm{II}_{1}$ systems $(X, \Gamma, \mu)$ have AC entropy 1.

PROPOSITION 4.3. For all $c,(0 \leq c \leq 1)$ there exists a $\operatorname{III}_{1}$ system $(X, \Gamma, \mu)$ with $h_{\mathrm{AC}}(\mu)=c$.

PROOF. (i) $h_{\mathrm{AC}}(\mu)=0$. We take an example from [5].

EXAMPLE. Let $\left\{\sigma_{i}=\log \left(1+a_{i}\right) /\left(1-a_{i}\right)\right\}_{i=1}^{\infty}$ be a sequence of the form

$$
\rho_{1}, \rho_{1}, \ldots, \rho_{1}, \rho_{2}, \rho_{2}, \ldots, 2 \rho_{1}, 2 \rho_{1}, \ldots, 2 \rho_{2}, 2 \rho_{2}, \ldots,
$$

where $\rho_{1} / \rho_{2} \notin \mathbb{Q}$ and if $n_{k_{i}}$ is the number of terms $k \rho_{i}$ then $n_{k_{i}} \geq e^{k \rho_{i}}$.

Now it can be shown that $e^{-\rho_{1}}$ and $e^{-\rho_{2}}$ are both in the ratio set of this measure and hence that $\mu$ is a type $\mathrm{III}_{1}$ measure where $a_{i} \rightarrow 1$ and so $h_{\mathrm{AC}}(\mu)=0$.

(ii) $h_{\mathrm{AC}}(\mu)=1$. For $h_{\mathrm{AC}}(\mu)=1$ we merely quote [3, Proposition 6.2]: 'If $a_{i} \rightarrow 0$ and $\sum a_{i}^{2}=\infty$ then $\mu$ is of type III $_{1}$.'

(iii) $h_{\mathrm{AC}}(\mu)=c, 0<c<1$. We can easily obtain a $\mathrm{III}_{1}$ system by defining

$$
\begin{array}{llll}
\mu_{i}(0)=(1-a) / 2, & \mu_{i}(1)=(1+a) / 2 & \text { for } i \text { odd } & (0<a<1), \\
\mu_{i}(0)=(1-b) / 2, & \mu_{i}(1)=(1+b) / 2 & \text { for } i \text { even } & (0<b<1),
\end{array}
$$

where

$$
\frac{\log ((1+a) /(1-a))}{\log ((1+b) /(1-b))} \notin \mathbb{Q}
$$

Define $\mu=\bigotimes_{i=1}^{\infty} \mu_{i}$ so that

$$
H\left(\mu_{i}\right)= \begin{cases}-\frac{1+a}{2} \log \frac{1+a}{2}-\frac{1-a}{2} \log \frac{1-a}{2}=c+\delta, & \text { for } i \text { odd } \\ -\frac{1+b}{2} \log \frac{1+b}{2}-\frac{1-b}{2} \log \frac{1-b}{2}=c-\delta, & \text { for } i \text { even, }\end{cases}
$$

where $\delta,(0<\delta<c)$, is such that

$$
f(\delta)=\frac{\log ((1+a(\delta)) /(1-a(\delta)))}{\log ((1+b(\delta)) /(1-b(\delta)))} \notin \mathbb{Q} .
$$

(The function $f$ decreases continuously from 1 so such a $\delta$ must exist.) Hence $h_{\mathrm{AC}}(\mu)=1 / 2(c+\delta)+1 / 2(c-\delta)=c$. 
PROPOSITION 4.4. Fix $\lambda,(0 \leq \lambda<1)$. For any $c,(0 \leq c \leq 1)$, there exists $a$ III $_{\lambda}$ system $(X, \Gamma, \mu)$ such that $h_{\mathrm{AC}}(\mu)=c$.

PROOF. (a) Case $\mathrm{III}_{\lambda},(0<\lambda<1)$.

(i) $h_{\mathrm{AC}}(\mu)=0$. Define $\mu=\bigotimes_{i=1}^{\infty} \mu_{i}$ in blocks of integer length $n_{j} \geq 1 / \lambda^{j}$, $j=1,2, \ldots$, so that for $i$ in the $j$ th block $\mu_{i}(0)=\lambda^{j} /\left(\lambda^{j}+1\right), \mu_{i}(1)=1 /\left(\lambda^{j}+1\right)$. As $i \rightarrow \infty, H\left(\mu_{i}\right) \rightarrow 0$ so $h_{\mathrm{AC}}(\mu)=0$.

(ii) $h_{\mathrm{AC}}(\mu)=1$. For each $n \in \mathbb{N}$ let $\Lambda(n) \subset[0, n]$ be an index set such that $(1 / 2)[\sqrt{n}] \leq \# \Lambda(n) \leq[\sqrt{n}]$. For $i \in \Lambda(n)$, let $\mu_{i}(0)=v^{\lambda}(0)=\lambda /(1+\lambda)$, $\mu_{i}(1)=v^{\lambda}(1)=1 /(1+\lambda)$, while for $i \in[0, n] \backslash \Lambda(n)$ let $\mu_{i}(0)=\mu_{i}(1)=1 / 2$. Then

$$
h_{\mathrm{AC}}(\mu)=\lim _{n \rightarrow \infty} \frac{n-\# \Lambda(n)}{n}+\frac{\# \Lambda(n)}{n} H\left(v^{\lambda}\right)=1 .
$$

(iii) $h_{\mathrm{AC}}(\mu)=c \in \mathbb{Q},(0<c<1)$. Let $c=m / n$, for $m, n \in \mathbb{Z}^{+}$. We define the required measure by taking the sequence $\sigma_{i}=\left\{\log \left(1+a_{i}\right) /\left(1-a_{i}\right)\right\}=\left\{-\log \lambda^{j(i)}\right\}$ from the measure constructed in part (i) and inserting strings of 0 values to form a new sequence $\sigma_{i}^{\prime}$. Take a block of length $n$. In the first $m$ places we put $\sigma_{i}^{\prime}=0$, that is, $H\left(\mu_{i}\right)=1$. In the remaining places put $\sigma_{m+i}^{\prime}=\sigma_{i}=\left\{-\log \lambda^{j(i)}\right\}$ for $i=1,2, \ldots, n-m$.

Repeat the process, taking another $m$ terms with $\sigma_{i}^{\prime}=0$ and then the next $n-m$ values in the $\left\{-\log \lambda^{j(i)}\right\}$ sequence. Continuing in this manner the resultant measure $\mu$ will be of type III by Moore's Criteria as there will still be $n_{k}$ terms with $1-a_{i}=$ $2 \lambda^{k} / 1+\lambda^{k}$, and further $\mu$ will be of type $\mathrm{III}_{\lambda}$ as we have not changed the ratio set. It is clear that $h_{\mathrm{AC}}(\mu)=m / n$.

(iv) $h_{\mathrm{AC}}(\mu)=c \notin \mathbb{Q},(0<c<1)$. It is easily seen that by using rational approximations we can adapt the measure from (iii) to provide examples of III $_{\lambda}$ systems with irrational AC entropies.

Take, for example, the decimal expansion of $c=d_{1} / 10+d_{2} / 10^{2}+d_{3} / 10^{3}+\cdots \equiv$ $d_{1} d_{2} d_{3} \ldots$ where $d_{i} \in\{0,1, \ldots, 9\}$. Choose $\epsilon(0<\epsilon<1)$. Let $c_{i}$ be a series of decimal approximations to $c$ with $c_{i}<c_{i+1}$ for all $i \in \mathbb{N}$ so that $c-c_{i}<\epsilon^{i} / 2$. First define the $\mu_{i}$ in blocks of length $10^{n_{1}}$ where $c_{1}=d_{1} d_{2} \cdots d_{n_{1}}$. The first $d_{1} d_{2} \cdots d_{n_{1}}$ places will have $H\left(\mu_{i}\right)=1$, while the remaining $\mu_{i}$ will be taken from the sequence defined by the $\sigma_{i}$ sequence used in part (iii).

Choose $N_{1}$ so that if this cycle were repeated infinitely, then for all $n>N_{1}$ we would have $\left|H\left(\beta_{n}\right) / n-c_{1}\right|<\epsilon / 2$. After that, keep defining $\mu_{i}$ in blocks of length $10^{n_{1}}$, but now make sure that $d_{1} d_{2} \cdots d_{n_{2}}$ of each $10^{n_{2}}$ places has $H\left(\mu_{i}\right)=1$. Then choose $N_{2}$ so that for all $n>N_{2}$ we would have $\left|H\left(\beta_{n}\right) / n-c_{2}\right|<\epsilon^{2} / 2$ and so on.

Now $c_{n} \rightarrow c$ as $n$ increases and further after each $N_{k},\left|H\left(\beta_{n}\right) / n-c\right|<2 \epsilon^{k}$.

(b) Case $\mathrm{III}_{0}$. 
(i) $h_{\mathrm{AC}}(\mu)=0$. Here we refer to an example from [5]. We shall modify it in parts (ii), (iii) and (iv).

EXAMPLE. Let $\left\{\sigma_{i}\right\}_{i=1}^{\infty}$ be of the form

$$
\ldots, \underbrace{2^{k}, 2^{k}, \ldots, 2^{k}}_{n_{k} \text { terms }}, \underbrace{2^{k+1}, \ldots, 2^{k+1}}_{n_{k+1} \text { terms }}, 2^{k+2}, \ldots
$$

To ensure that $\mu$ is of type III we choose the $n_{k}$ to satisfy part (3) of Moore's Criteria so that $\sum_{i=1}^{\infty}\left(1-a_{i}\right)=\infty$. Here we have $1-a_{i}=2 /\left(1+e^{\sigma_{i}}\right)$ so we can take $n_{k} \geq\left(e^{2^{k}}+1\right) / 2$. The resulting measure $\mu$ is of type $\mathrm{III}_{0}$. Further, $\lim _{i \rightarrow \infty} a_{i}=1$ so we have $h_{\mathrm{AC}}(\mu)=0$.

(ii) $h_{\mathrm{AC}}(\mu)=1$. We modify the sequence $\left\{\sigma_{i}\right\}$ from part (i) by inserting long sequences of zeroes (that is, $\mu_{i}$ with entropy 1 ) between the $2^{k}$ strings; that is,

$$
\underbrace{2^{k}, 2^{k}, \ldots, 2^{k}}_{n_{k} \text { lerms }}, \underbrace{0,0, \ldots, 0}_{N_{k} \text { terms }}, \underbrace{2^{k+1}, \ldots, 2^{k+1}}_{n_{k+1} \text { lerms }}
$$

where the $N_{k}$ are chosen so large that $\sum_{j=1}^{k+1} n_{j}<\sqrt{N_{k}}$.

Our new measure is still type $\mathrm{III}_{0}$ but we now have $h_{\mathrm{AC}}(\mu)=1$.

(iii) $h_{\mathrm{AC}}(\mu)=c \in \mathbb{Q},(0<c<1)$. Let $c=m / n$, for $m, n \in \mathbb{Z}^{+}$. Again we will take our $\mathrm{III}_{0}$ measure from part (i) and add strings of zeroes to the sequence $\left\{\sigma_{i}\right\}_{i=1}^{\infty}$.

Define $\left\{\sigma_{i}^{\prime}\right\}_{i=1}^{\infty}$ in blocks of length $n$. The first $m$ values for $\sigma_{i}^{\prime}$ in each block will be zero, that is, the $\mu_{i}$ have entropy 1 . The remaining $n-m$ values in the block will be taken from the original $\left\{\sigma_{i}\right\}$ sequence and integrated into the $\left\{\sigma_{i}^{\prime}\right\}$ sequence with their order undisturbed, for example,

$$
\overbrace{\underbrace{0,0, \ldots, 0}_{n}, 2^{k}, 2^{k}, 2^{k+1}, \ldots, 2^{k+1}}^{m} .
$$

There will still be $n_{k}$ terms of $2^{k}$ so our resulting measure will still be type III, and as we lave not changed the ratio set we know it will be type $\mathrm{III}_{0}$. Clearly $h_{\mathrm{AC}}(\mu)=m / n=c$ is required.

(iv) $h_{\mathrm{AC}}(\mu)=c \notin \mathbb{Q},(0<c<1)$. We obtain $\mathrm{III}_{0}$ systems with irrational $\mathrm{AC}$ sntropies by modifying the measure from part (iii) using rational approximations, as llustrated previously in the $\mathrm{III}_{\lambda}$ case.

\section{IC equivalence}

In this section we introduce an equivalence relation-Initial Co-ordinates or IC :quivalence-for which AC entropy is an invariant. The definition of this equivalence 
relation was inspired by Proposition 2.3. Although that result dealt solely with permuted product measures, the formulation of IC equivalence contains no such restriction.

DEFINITION 5.1. Let $(X, \Gamma, \mu)$ and $(X, \Gamma, v)$ be two systems of finite co-ordinate changes. Suppose we have an invertible, bi-measurable map $\phi:(X, \mu) \rightarrow(X, \nu)$ satisfying the following: For each $n \in \mathbb{N}$ there exist $m_{1}(n), m_{2}(n) \in \mathbb{N}$ with

$$
m_{1}(n) \leq n \leq m_{2}(n), \quad m_{1}(n) \leq m_{1}(n+1), \quad m_{2}(n) \leq m_{2}(n+1),
$$

and

$$
\lim _{n \rightarrow \infty} \frac{m_{1}(n)}{n}=\lim _{n \rightarrow \infty} \frac{m_{2}(n)}{n}=1,
$$

such that for almost all $x, y \in X$,

(i) if $\phi(x)$ and $\phi(y)$ have the same first $n$ co-ordinates (that is, they are initially equal), then $x$ and $y$ have the same first $m_{1}(n)$ co-ordinates; and

(ii) if $x$ and $y$ have the same first $m_{2}(n)$ co-ordinates, then $\phi(x)$ and $\phi(y)$ have the same first $n$ co-ordinates.

Now if $\mu \circ \phi=\nu\left(\right.$ here $\mu \circ \phi(E)=\mu\left(\phi^{-1}(E)\right)$ for measurable $\left.E\right)$ we say $(X, \Gamma, \mu)$ and $(X, \Gamma, \nu)$ are IC equivalent.

NOTE. Our definition requires that $\mu \circ \phi=\nu$. It would be preferable to weaken this requirement to $\mu \circ \phi \sim \nu$ as is the case for orbit equivalence. Remember that our aim is to develop an equivalence relation for which $\mathrm{AC}$ entropy is an invariant. In certain cases we could replace the measure equality condition with measure equivalence and still achieve our objective. For example, if $\mu, \mu \circ \phi$ and $v$ were all product measures then, as our working will later show, all that would be required to establish $h_{\mathrm{AC}}(\mu)=h_{\mathrm{AC}}(\nu)$ would be $\mu \circ \phi \sim v$ for a suitable $\phi$. However as we have shown in Section 3, this may not be sufficient in the general case.

\section{LEMMA 5.2. IC equivalence is an equivalence relation.}

PROOF. Reflexivity is obvious, taking $\phi$ as the identity map.

For symmetry, suppose $\mu$ is IC equivalent to $v$ via the map $\phi$, with sequences $m_{1}(n)$ and $m_{2}(n)$ to guarantee properties (i) and (ii) in our definition. We will show that for the map $\phi^{-1}$ we can find $m_{1}^{\prime}(n)$ and $m_{2}^{\prime}(n)$ for each $n$ to satisfy these same properties.

Let $x$ and $y$ have the same first $n$ co-ordinates. We want $m_{1}^{\prime}(n) \leq n$ such that $\phi(x)$ and $\phi(y)$ have the same first $m_{1}^{\prime}(n)$ co-ordinates.

We know from property (ii) that if $x$ and $y$ have the same first $m_{2}(p)$ co-ordinates then $\phi(x)$ and $\phi(y)$ have the same first $p$ co-ordinates. So if $n=m_{2}(p)$ for some $p \in \mathbb{N}$, we could take $m_{1}^{\prime}(n)=p$. Let $p \in \mathbb{N}$ be the greatest integer such that 
consider the partitioning of orbit equivalence classes by IC equivalence. Establishing an intermediate classification between measure equivalence and orbit equivalence could yield some interesting results. In fact such a scheme may even be necessary if we are ever to gain a clearer picture of the $\mathrm{III}_{0}$ class.

\section{Acknowledgements}

This work forms part of the author's PhD thesis. The author wishes to thank Tony Dooley, Dan Rudolph, Jane Hawkins and Toshihiro Hamachi for their comments and suggestions and the Australian Research Council for their financial support.

\section{References}

[1] G. Brown and A. H. Dooley, 'Odometer actions on $G$-measures', Ergodic Theory Dynam. Systems 11 (1991), 279-307.

[2] —_, 'Dichotomy theorems for G-measures', Internat. J. Math. 5 (1994), 827-834.

[3] G. Brown, A. H. Dooley and J. Lake, 'On the Krieger-Araki-Woods ratio set', Tohoku Math. J. (2) 47 (1995), 1-13.

[4] J. L. Doob, Stochastic processes (Wiley, New York, 1953).

[5] A. H. Dooley, I. Klemes and A. N. Quas, 'Product and Markov measures of type III', J. Austral. Math. Soc. Ser.A 65 (1998), 84-110.

[6] M. Keane, 'Strongly mixing g-measures', Invent. Math. 16 (1972), 309-324.

[7] R. LePage and V. Mandrekar, 'On likelihood ratios of measures given by Markov chains', Proc. Amer. Math. Soc. 52 (1975), 377-380.

[8] A. A. Lodkin, 'Absolute continuity of measures corresponding to Markov processes with discrete time', Theory Probab. Appl. 16 (1971), 690-694.

[9] C. Moore, 'Invariant measures on product spaces', in: Proc. Fifth Berkeley Sympos. Math. Statist. and Probability (Berkeley, Calif., 1965/66) Vol. II: Contributions to probability theory, Part 2 (Univ. California Press, Berkeley, 1966) pp. 447-459.

[10] G. Mortiss, 'An invariant for non-singular isomorphism', Ergodic Theory Dynam. Systems (submitted).

[11] - Average co-ordinate entropy and a non-singular version of restricted orbit equivalence (Ph.D. Thesis, University of New South Wales, 1997).

[12] C. E. Silva and P. Thieullen, 'A skew product entropy for non-singular transformations', J. London Math. Soc. (2) 52 (1995), 497-516.

School of Mathematics

The University of New South Wales

Sydney NSW 2052

Australia

e-mail: mortiss@maths.unsw.edu.au 